\title{
Application of Clebsch-Gordan Coefficients and Isomorphic Frame Transformations to Invert Earth's Changing Geometrical Shape for Continental Hydrological Loading and Sea Level's Passive Response
}

\author{
${ }^{1,2}$ Geoffrey Blewitt, ${ }^{2}$ Peter Clarke, ${ }^{1}$ David Lavallée and ${ }^{2}$ Konstantin Nurutdinov \\ ${ }^{1}$ Nevada Bureau of Mines and Geology, and Seismological Laboratory, University of Nevada, Reno, U.S.A. \\ ${ }^{2}$ School of Civil Engineering and Geosciences, University of Newcastle, Newcastle upon Tyne, U.K.
}

\begin{abstract}
The hydrological cycle induces mass exchange between the oceans and continents, and redistribution of water within the continents. In turn, relative sea level passively responds to the change in shape of both the geoid and the ocean bottom. The time variation in these two surfaces must self-consistently relate to the total mass redistribution. Here we show formally how, if given a time series of station coordinates from a global network of geodetic stations, we can invert for the separate contributions to the total mass redistribution due to water on the continents and water in the oceans. If we characterize the deformation of the solid Earth in terms of a truncated vector spherical harmonic expansion, the solution to this problem can be formulated in terms of Clebsch-Gordan coefficients, which are familiar for the calculation of branching ratios in elementary particle physics. As part of a rigorous solution to this problem, we formally address the definition of reference frame by applying the concept of an "isomorphic frame transformation," which conserves the formal theoretical relationship between surface deformation and load distribution by a simultaneous translation of origin and change of degree-one load Love numbers. We show explicitly how the solution to the inversion problem is derived and implemented, and we present an example of this procedure in practice using real GPS data from the IGS network.
\end{abstract}

Keywords. Clebsch-Gordan, isomorphic, hydrological cycle, frame transformation, Love number

\section{Introduction}

We have developed a methodology to invert for the redistribution of fluids on the Earth's surface given precise global geodetic measurements of Earth's geometrical shape [Blewitt and Clarke, 2003]. Three steps are involved: (1) inversion of geodetic station coordinates for a spherical harmonic representation of Earth's shape; (2) inversion of Earth's shape for surface mass distribution; (3) inversion for a specific surface mass distribution consistent with static ocean equilibrium theory.

Here we focus on the methodology of step (3), which is facilitated by the use of Clebsch-Gordan (CG) coefficients. The CG method is applied where it is appropriate to spatially partition a spherical function into two spatial domains (land and ocean) which are characterized by different properties (such as the land's ability to concentrate water).

Secondly, we highlight our findings on reference frame theory, showing how to transform loading equations between frames [Blewitt, 2003].

\section{Clebsch-Gordan Coefficients}

\subsection{Spectral Model for Function on Sphere}

An arbitrary function on the sphere $f(\Omega)$ at position $\Omega$ (latitude $\varphi$, longitude $\lambda$ ) can be expanded:

$$
f(\Omega)=\sum_{n=0}^{\infty} \sum_{m=-n}^{n} f_{n m} Y_{n m}(\Omega)
$$

where the complex spherical harmonics are expressed using associated Legendre polynomials:

$$
\begin{aligned}
Y_{n m}(\Omega) & =\sqrt{\frac{2 l+1}{4 \pi} \frac{(n-m) !}{(n+m) !}} P_{n m}(\sin \phi) e^{i m \lambda} \\
& =(-1)^{m} Y_{n(-m)}^{*}(\Omega)
\end{aligned}
$$

where

$$
\oiint Y_{n m}(\Omega) Y_{n^{\prime} m^{\prime}}^{*}(\Omega) d \Omega=\delta_{n n^{\prime}} \delta_{m m^{\prime}}
$$

Therefore coefficients in equation (1) for a real- 
valued function are given by

$$
f_{n m}=\oiint f(\Omega) Y_{n m}^{*} d \Omega=(-1)^{m} f_{n(-m)}^{*}(\Omega)
$$

\subsection{Spectral Model for Multiple Functions}

Consider the case where it is appropriate to express

$$
f(\Omega)=\tilde{f}(\Omega) c(\Omega)
$$

We shall be interested in the case where $\tilde{f}(\Omega)$ is a function that varies (with choice of model, epoch, or data set) and is represented as a truncated expansion to degree $\bar{n}$, and $c(\Omega)$ is a constant function (that does not change with scenario) known to any arbitrary degree and order. In the language of signal processing, we might consider $\tilde{f}(\Omega)$ as the signal and $c(\Omega)$ the filter. Applying equation (4):

$$
\begin{aligned}
f_{n m} & =\oiint \tilde{f}(\Omega) c(\Omega) Y_{n m}^{*}(\Omega) d \Omega \\
f_{n m} & =\sum_{n^{\prime}, m^{\prime}}^{\bar{n}} \tilde{f}_{n^{\prime} m^{\prime}}\left(\sum_{n^{\prime \prime}, m^{\prime \prime}}^{2 n} c_{n^{\prime \prime} m^{\prime \prime}} Q_{n^{\prime} m^{\prime} ; n^{\prime \prime} m^{\prime \prime} ; n m}\right)
\end{aligned}
$$

where we have chosen to first sum over the coefficients of the constant function $c(\Omega)$, and where

$$
Q_{n^{\prime} m^{\prime} ; n^{\prime \prime} m^{\prime \prime} ; n m} \equiv \oiint Y_{n^{\prime} m^{\prime}}(\Omega) Y_{n^{\prime \prime} m^{\prime \prime}}(\Omega) Y_{n m}^{*}(\Omega) d \Omega
$$

Particle physics and the theory of atomic spectra enjoy broad application of equation (7), which can be computed in terms of tabulated CG coefficients (in angled brackets) [Particle Data Group, 2002]:

$$
\begin{aligned}
Q_{n^{\prime} m^{\prime} ; n^{\prime \prime} m^{\prime \prime} ; n m} & =\sqrt{\frac{\left(2 n^{\prime}+1\right)\left(2 n^{\prime \prime}+1\right)}{4 \pi(2 n+1)}} \\
& \times\left\langle n^{\prime}, m^{\prime} ; n^{\prime \prime}, m^{\prime \prime} \mid n, m\right\rangle\left\langle n^{\prime}, 0 ; n^{\prime \prime}, 0 \mid n, 0\right\rangle
\end{aligned}
$$

\subsection{Selection Rules}

The general result of equation (8) is zero unless the following well-known "selection rules" are obeyed:

$$
\begin{gathered}
m=m^{\prime}+m^{\prime \prime} \\
\left|n^{\prime}-n^{\prime \prime}\right| \leq n \leq n^{\prime}+n^{\prime \prime} \\
n+n^{\prime}+n^{\prime \prime}=\text { even }
\end{gathered}
$$

These rules significantly limit the number of summation terms in equation (6). "Selection rules" are fundamental in quantum phenomena as they specify which possible outcomes of an interaction are allowed or forbidden. Such rules derive from the properties of spherical harmonics. For example, the multiple of two pure degree- 2 spherical harmonics is a linear combination of only degrees 0,2 , and 4 .

A useful consequence of selection rules for our purposes is that $c(\Omega)$ need only be expressed to degree $2 n$ to get an exact result in equation (6). This fact can be exploited, for example, if we associate $c(\Omega)$ with the ocean function, which is defined to be one over the oceans and zero on land, and is therefore discontinuous on coastlines:

$$
c(\Omega) \equiv\left\{\begin{array}{l}
1(\Omega \in \text { ocean }) \\
0(\Omega \in \text { land })
\end{array}\right.
$$

Ocean function coefficients are computed using coastline data [Balmino et al., 1973]. It may be counter-intuitive that the ocean function can be accounted for exactly in equation (6) even though in principle such a discontinuous function is poorly represented (alone) by a truncated expansion.

\subsection{Product-to-Sum Operator}

Given that $c(\Omega)$ is independent of scenario, it is convenient in equation (6) to first tabulate (once and for all) the following matrix of coefficients:

$$
C_{n m ; n^{\prime} m^{\prime}}=\sum_{n^{\prime \prime}, m^{\prime \prime}}^{2 n} c_{n^{\prime \prime} m^{\prime \prime}} Q_{n^{\prime} m^{\prime} ; n^{\prime \prime} m^{\prime \prime} ; n m}
$$

for all $n^{\prime} \leq \bar{n}$ and all $n$ of potential interest. Once tabulated, equation (6) becomes the matrix equation

$$
\begin{aligned}
f_{n m} & =\sum_{n^{\prime}, m^{\prime}}^{\bar{n}} C_{n m ; n^{\prime} m^{\prime}} \tilde{f}_{n^{\prime} m^{\prime}} \\
\mathbf{f} & =\mathbf{C} \tilde{\mathbf{f}}
\end{aligned}
$$

where matrix $\mathbf{C}$ is the "product-to-sum operator." Thus the problem of multiplying two functions in the spatial domain has been converted to a matrix multiplication of spectral coefficients. In the case that $c(\Omega)$ is the ocean function, equation (12) computes the filtering effect of the ocean mask on the spectral coefficients. This allows relative sea level to be represented as a lower-degree spherical har- 
monic function than would otherwise be required to account for the coastlines.

\section{Spatial Representation Model}

\subsection{Naïve Mapping}

Consider the general situation for a function $f(\Omega)$ we have determined by some experimental means the spectral coefficients up to degree and order $\bar{n}$, and we then wish to map this function on the sphere. In this case the "naïve" spatial representation of $f(\Omega)$ is a truncated spherical harmonic expansion:

$$
f(\Omega) \cong \bar{f}(\Omega)=\sum_{n, m}^{\bar{n}} f_{n m} Y_{n m}(\Omega)
$$

\subsection{Spatial Domain Partitioning}

Suppose we know a priori that $f(\Omega)$ is discontinuous at coastlines, and perhaps we have a model that distinguishes its behavior separately over the ocean and on land. It is better to incorporate the $a$ priori information by partitioning the spatial representation between the ocean $s(\Omega)$ and land $l(\Omega)$ :

$$
\begin{aligned}
f(\Omega) & =s(\Omega)+l(\Omega) \\
& =c(\Omega) \tilde{s}(\Omega)+[1-c(\Omega)] \tilde{l}(\Omega)
\end{aligned}
$$

where $c(\Omega)$ is the ocean function, and where the tildes denote functions that are smooth with no discontinuity at the coastlines.

Now we can apply the product-to-sum conversion given by equation (12) to find the relationship between spectral coefficients of $f(\Omega)$ and the spectral coefficients of the smooth functions over ocean $\tilde{s}(\Omega)$ and land $\tilde{l}(\Omega)$ :

$$
\begin{aligned}
f_{n m}= & \sum_{n^{\prime}, m^{\prime}} C_{n m ; n^{\prime} m^{\prime}} \tilde{s}_{n^{\prime} m^{\prime}} \\
& +\sum_{n^{\prime}, m^{\prime}}\left(\delta_{n n^{\prime}} \delta_{m m^{\prime}}-C_{n m ; n^{\prime} m^{\prime}}\right) \tilde{l}_{n^{\prime} m^{\prime}} \\
\mathbf{f} & =\mathbf{C} \tilde{\mathbf{s}}+(\mathbf{I}-\mathbf{C}) \tilde{\mathbf{l}}
\end{aligned}
$$

Equation (15) is the spectral equivalent to equation (14). For example, given low-degree approximations to land and ocean loading models, equation (15) can be used to compute the spectrum of the total load accounting for effect of the coastline discontinuity. The spatial equivalent to this computation would be to take equation (14) and apply a Green's function convolution [Wahr, 1982]. There are advantages to the spectral approach, however, when investigating long-wavelength phenomena, or for constructing an invertible system of equations to estimate low-degree loading coefficients directly from observational data. For example, equation (15) may be more convenient for studying the effect of degree-2 loading on Earth rotation, or degree-1 loading on geocenter motion.

\subsection{Physical Constraints}

Consider the case where the smooth functions over ocean and land are functionally related through the physics of the problem (e.g., through the shape of the geoid and the shape of the deformed Earth). If $a$ priori physical constraints can be applied such that the number of free variables are equal on both sides of equation (15), it then becomes possible to invert this for ocean and land functions truncated at degree $\bar{n}$. In Section 4 we give concrete examples of how to apply such physical constraints.

\subsection{Partitioned Spatial Representation}

The last step is to then map these smooth functions only over their respective domains, that is with discontinuities at the coastlines.

$$
f(\Omega) \cong \hat{f}(\Omega)=\sum_{n, m}^{\bar{n}}\left\{\begin{array}{l}
\tilde{s}_{n m} Y_{n m}(\Omega \in \text { ocean }) \\
\tilde{l}_{n m} Y_{n m}(\Omega \in \text { land })
\end{array}\right.
$$

Equation (15) guarantees that the coefficients of $f(\Omega)$ are consistent with the original truncated expansion, but in effect, the spatial solution (16) augments the truncated expansion to infinite degree and order so as to be consistent with the physical relationship between ocean and land functions. Equation (15) is useful in that it allows the presence of coastlines to dominate the spectrum of $f(\Omega)$ at higher degrees (rather than short wavelength variability within the two spatial domains).

\section{Application to Hydrological Loading}

\subsection{Physical Constraint: Sea Level Given the Total Load}

We have applied the above concepts to the problem of partitioning the loading of water associated with 
the hydrological cycle between ocean and land. Here we apply a static oceanographic model to derive a set of physical constraints that can be used to reduce the number of free parameters in the problem. Over the ocean the water load can be parameterized as relative sea level, and over land it can be considered as an equivalent thickness of water.

We assume:

1. redistribution of water on land is arbitrary;

2. the total mass of surface water is conserved, $f_{00}=0$;

3. the sea surface is an equipotential, which generally does not equate to the (deformed) geoid;

4. the ocean bottom deforms with the solid Earth;

5. the solid Earth deforms in response to the spectral coefficients of the total load according to load Love number theory [Farrell, 1972].

The weakest assumption is number three, in that the ocean has dynamic topography due to ocean currents that may vary. For purposes of demonstrating the technique, however, ocean topography is assumed to be in static equilibrium. This is in any case instructive to compute the effect of land loading on relative sea level, which to first order might be considered uncoupled to ocean currents.

Under these conditions it can be shown [Blewitt and Clarke, 2003] that there is a linear relationship between the spectral coefficients of relative sea level and the total load for degrees other than zero:

$$
\begin{aligned}
& \tilde{s}_{n m}=\varsigma_{n} f_{n m} \quad \text { for } n \geq 1 \\
& \varsigma_{n} \equiv \frac{3\left(1+k_{n}-h_{n}\right) \rho_{S}}{(2 n+1) \rho_{E}}
\end{aligned}
$$

where $\rho_{E}=5514 \mathrm{~kg} \mathrm{~m}^{-3}$ is the mean density of the Earth, $\rho_{S}=1025 \mathrm{~kg} \mathrm{~m}^{-3}$ is the density of seawater; the degree- $n$ load Love numbers are $k_{n}$ for the gravitational potential and $h_{n}$ for surface height of the solid Earth (not to be confused with tidal Love numbers). The first term $1+k_{n}$ in the numerator of equation (17) relates to the sea surface taking on the shape of the deformed geoid, and the last term $-h_{n}$ accounts for the change in relative height of the sea surface above the deformed ocean basin.

In contrast, the degree- 0 term of relative sea level is not governed by the degree- 0 term of the total load, which vanishes by conservation of mass $f_{00}=0$, but rather relates to the balance between the volume of the ocean basin enclosed by the deformed geoid, and the water available to fill that basin:

$$
\tilde{s}_{00}=\sum_{n^{\prime}, m^{\prime}}^{n}\left[1-\varsigma_{n^{\prime}}\right] \frac{c_{n^{\prime} m^{\prime}} f_{n^{\prime} m^{\prime}}}{c_{00}}
$$

where the first term in the square bracket is related to change in global mean sea level from mass exchange with the land, and the smaller second term [Dahlen, 1976] is the contribution of the geoidcapped basin volume, which is generally non-zero even in the absence of mass exchange.

Equations (17) and (18) may be combined and expressed in matrix form:

$$
\widetilde{\mathbf{s}}=\mathbf{B f}
$$

where $\mathbf{B}$ is a square $(n \times n)$ matrix. Thus relative sea level can be computed up to the same degree and order as for the total load.

\subsection{Total Load Given the Land Load}

The total load is related to the smooth land load coefficients by application of the physical constraints given by equation (19) to the general partitioned spectral model, equation (15):

$$
\begin{gathered}
\mathbf{f}=\mathbf{C B f}+(\mathbf{I}-\mathbf{C}) \tilde{\mathbf{l}} \\
(\mathbf{I}-\mathbf{C B}) \mathbf{f}=(\mathbf{I}-\mathbf{C}) \tilde{\mathbf{l}}
\end{gathered}
$$

Given a spectral model of the land load $\tilde{\mathbf{l}}$ to degree $\bar{n}$ we can invert for the spectral model of the total load to any desired degree $n$

$$
\mathbf{f}=(\mathbf{I}-\mathbf{C B})^{-1}(\mathbf{I}-\mathbf{C}) \tilde{\mathbf{I}}
$$

which is well-conditioned, due fundamentally to the small ratio of the density of water to that of the Earth. This specific application of Clebsch-Gordan coefficients might be used, for example, to compute the extra contribution of the ocean to predictions of geocenter motion or polar motion from continental hydrology. In effect, the ocean acts to amplify the signal caused by land loading through equation (21).

\subsection{Partitioned Load Given the Total Load}

Alternatively, we might already have an estimate of the spectral coefficients of the change in total surface load based on the geodetically measured change in the Earth's shape or gravity field. In this case, the spectral model of the partitioned load is computed in two steps by equations (19) and (15): 


$$
\begin{aligned}
& \tilde{\mathbf{s}}=\mathbf{B f} \\
& \tilde{\mathbf{l}}=(\mathbf{I}-\mathbf{C})^{-\mathbf{1}}(\mathbf{f}-\tilde{\mathbf{s}})
\end{aligned}
$$

The maximum degree of partitioned models $\tilde{\mathbf{s}}$ and $\tilde{\mathbf{l}}$ is that of the given total load $\mathbf{f}$.

\subsection{Demonstration using Real Data}

Figure 1 shows an example of our solution using GPS data for partitioned ocean-land loads given an initial naïve model, equation (12) up to degree and order 2. As a rule, we find that the ocean load follows a spatially similar pattern as the land load, except that it is reduced an order of magnitude. Not applying the partitioning method might therefore greatly overestimate the ocean load near coastlines.
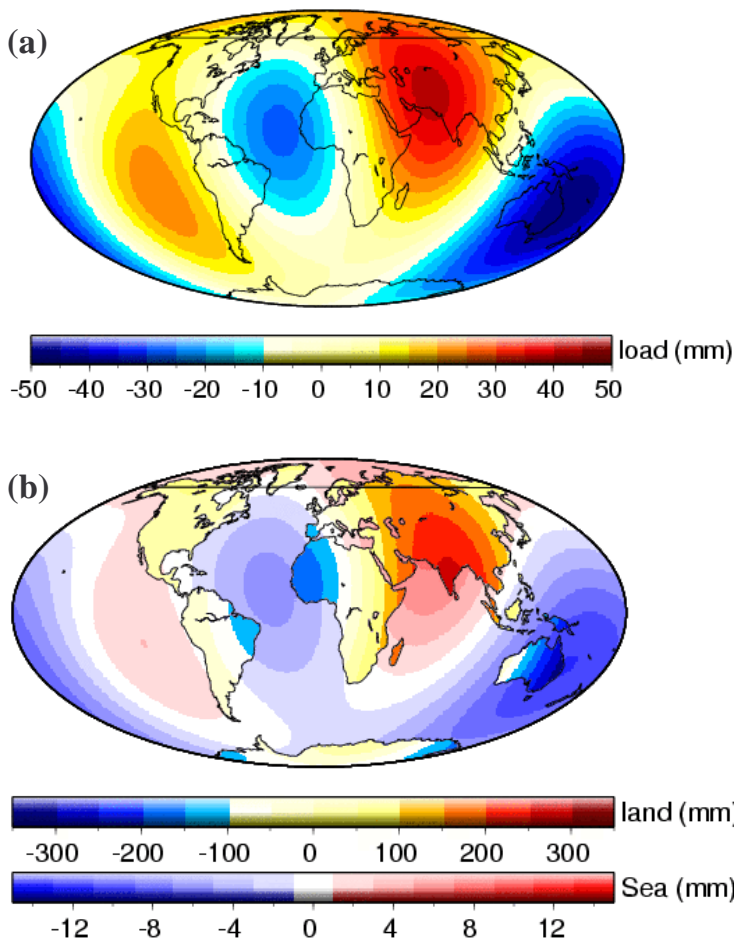

Fig. 1 Example of spatial partitioning of a global load function: (a) naïve spatial representation using a simple truncated spherical harmonic expansion; (b) partitioned function, applying physical constraints on static ocean topography.

\section{Isomorphic Frames}

The previous development assumes that we already have an estimate of the spectral coefficients of the total surface load. Such estimates can derive from GPS measurements of variation in Earth's shape, in an appropriate reference frame that is consistent with the degree-1 load Love numbers. Here we highlight the theoretical development and findings of Blewitt [2003] and Blewitt and Clarke [2003].

A vector surface displacement function on a sphere can be expressed [Grafarend, 1986]:

$$
\mathbf{D}(\Omega)=\nabla \Psi(\Omega)+\nabla \times(\Gamma(\Omega) \hat{\mathbf{r}})+H(\Omega) \hat{\mathbf{r}}
$$

where $H(\Omega)$ is the height function, $\Psi(\Omega)$ is the "poloidal" function, $\Gamma(\Omega)$ is the "toroidal" function, and $\nabla$ is the surface gradient operator. For normal loading problems, the toroidal component is zero. Expanding (23) in terms of spherical harmonics, we find the degree- 1 term for the displacement function can be written as a vector equation:

$$
\mathbf{D}_{1}(\Omega)=\boldsymbol{\Psi}_{1}+\hat{\mathbf{r}}\left[\left(\mathbf{H}_{1}-\boldsymbol{\Psi}_{1}\right) \cdot \hat{\mathbf{r}}\right]
$$

where we define the spatially constant vectors $\boldsymbol{\Psi}_{1}=\left(\Psi_{11}^{C}, \Psi_{11}^{S}, \Psi_{10}^{C}\right)$ and $\mathbf{H}_{1}=\left(H_{11}^{C}, H_{11}^{S}, H_{10}^{C}\right)$, using notation for classical (real) spherical harmonics. The same degree-one deformation, as observed in a reference frame that has an origin translated by vector $-\Delta \mathbf{r}_{\mathbf{0}}$ with respect to the original frame, will have (primed) surface displacements:

$$
\begin{aligned}
\mathbf{D}_{1}^{\prime}(\Omega) & =\mathbf{D}_{1}(\Omega)+\Delta \mathbf{r}_{\mathbf{0}} \\
& =\boldsymbol{\Psi}_{1}^{\prime}+\hat{\mathbf{r}}\left[\left(\mathbf{H}_{1}^{\prime}-\boldsymbol{\Psi}_{1}^{\prime}\right) \cdot \hat{\mathbf{r}}\right]
\end{aligned}
$$

where

$$
\begin{aligned}
\boldsymbol{\Psi}_{1}^{\prime} & =\boldsymbol{\Psi}_{1}+\Delta \mathbf{r}_{\mathbf{0}} \\
\mathbf{H}_{1}^{\prime} & =\mathbf{H}_{1}+\Delta \mathbf{r}_{\mathbf{0}}
\end{aligned}
$$

This implies a perhaps surprising result: there exist two special reference frames in which either the horizontal displacements or the vertical displacements are zero for degree-1 deformation [Blewitt et al., 2001]. These are the CL (center of lateral figure) and $\mathrm{CH}$ (center of height figure) frames, respectively [Blewitt, 2003]. That the vertical degree1 displacements can be made zero by a simple translation proves that the model Earth retains a shape of constant radius under a degree-1 deformation, and hence retains a perfect spherical shape. However, if $\mathbf{H}_{1}^{\prime}=0$, in general $\boldsymbol{\Psi}_{1}^{\prime}=\boldsymbol{\Psi}_{1}-\mathbf{H}_{1} \neq 0$; so the surface is strained, as detected by GPS [Blewitt et al., 2001] and by very long baseline interferometry [Lavallée and Blewitt, 2002].

Degree-1 displacement vector functions (including the degree-1 geoid height vector $\mathbf{N}_{1}$ ) relate to degree-1 load Love numbers [Blewitt et al., 2001]: 


$$
\left(\mathbf{H}_{\mathbf{1}}, \boldsymbol{\Psi}_{\mathbf{1}}, \mathbf{N}_{\mathbf{1}}\right)=-\left(h_{1}, l_{1}, 1+k_{1}\right) \Delta \mathbf{r}_{\mathrm{CE}}
$$

where $\Delta \mathbf{r}_{\mathrm{CE}}$ is the "geocenter" displacement of the solid Earth center of mass CE relative to the stable center of mass of the entire Earth system CM:

$$
\Delta \mathbf{r}_{\mathrm{CE}}=-\frac{\rho_{S}}{\rho_{E}}\left(\begin{array}{lll}
f_{11}^{C} & f_{11}^{S} & f_{10}^{C}
\end{array}\right)^{T}
$$

Degree-1 load Love numbers themselves must be transformed if equation (27) is to be retained under a frame transformation, which requires that $\Delta \mathbf{r}_{0}$ be aligned with the geocenter displacement:

$$
\Delta \mathbf{r}_{0}=\alpha \Delta \mathbf{r}_{\mathrm{CE}}
$$

so that from equations (26), (27) and (29):

$$
\begin{aligned}
\left(\mathbf{H}_{1}^{\prime}, \boldsymbol{\Psi}_{1}^{\prime}, \mathbf{N}_{\mathbf{1}}^{\prime}\right) & =-\left(h_{1}^{\prime}, l_{1}^{\prime}, 1+k_{1}^{\prime}\right) \Delta \mathbf{r}_{\mathrm{CE}} \\
& =-\left(h_{1}, l_{1}, 1+k_{1}\right) \Delta \mathbf{r}_{\mathrm{CE}}+\Delta \mathbf{r}_{0} \\
& =-\left(h_{1}, l_{1}, 1+k_{1}\right)(1-\alpha) \Delta \mathbf{r}_{\mathrm{CE}}
\end{aligned}
$$

Hence we can conclude that degree-1 load Love numbers transform as:

$$
\left(h_{1}^{\prime}, l_{1}^{\prime}, 1+k_{1}^{\prime}\right)=\left(h_{1}, l_{1}, 1+k_{1}\right)(1-\alpha)
$$

We call such special frames "isomorphic frames", as theoretically these are the only reference frames in which the degree-1 loading equation takes on the form of equation (27). Fortunately several frames in current use are isomorphic, including the $\mathrm{CH}, \mathrm{CL}$, CM, CE, and CF frames [Blewitt, 2003]. Frames that are not isomorphic include those where individual station height velocities are constrained.

Most commonly realized in GPS is the CF frame, in which the average vector surface displacement is constrained to zero. However, modelers tend to present results in the CE frame. Equation (31) allows the degree-1 load Love numbers to be transformed from the $\mathrm{CE}$ to the $\mathrm{CF}$ frame. The isomorphic parameter can be calculated for specific frames where the origin is defined either geometrically (with respect to observed surface displacements) or physically. (with respect to center of mass) [Blewitt, 2003]. In this case the appropriate value for the isomorphic parameter $\alpha=-0.021$, which is a small correction due to the $\mathrm{CE}$ and $\mathrm{CF}$ frame being almost identical. However, much larger values are possible for other isomorphic transformations.

\section{Conclusions}

We have developed two methodological tools that are useful for imposing self-consistency when inverting geodetic data for changes in global surface loading. The first tool considers the surface load as the multiple of two functions, a problem that can be transformed into the spectral domain by using Clebsch-Gordan coefficients. The CG method is demonstrated by imposing physical constraints on static ocean topography onto an assumed spectral loading distribution, so as to produce a realistic spatial representation of the load. The CG method may find broader application in geodesy to any spherical function that can be expressed as the multiple of a signal function, and a second known function, such as a filter function or an a priori model function.

The second tool transforms degree-1 load Love numbers into isomorphic frames in common use. Frames that are not isomorphic do not obey Love number relations, and so should be avoided.

Acknowledgements. The research was funded in the USA by NSF Geophysics and NASA Solid Earth and Natural Hazards, and in the UK by NERC. Thanks to G. Balmino for ocean loading coefficients. IGS provided the GPS data.

\section{References}

Balmino, G., K. Lambeck, and W.M. Kaula, A spherical harmonic analysis of Earth's topography, Journ. Geophys. Res., 78, 478-481, 1973.

Blewitt, G. and P. Clarke, Inversion of Earth's changing shape to weigh sea level in static equilibrium with surface mass redistribution, Journ. Geophys. Res., 108 (B6), 2311, doi:10.1029/2002JB002290, 2003.

Blewitt, G., Self-consistency in reference frames, geocenter definition, and surface loading of the solid Earth, Journ. Geophys. Res., doi:10.1029/2002JB002082, 2003.

Blewitt, G., D. Lavallée, P. Clarke, and K. Nurutdinov, A new global mode of Earth deformation: Seasonal cycle detected, Science, 294, 2,342-2,345, 2001.

Dahlen, F.A., The passive influence of the oceans upon the rotation of the Earth, Geophys. Journ. Roy. Astron. Soc., 46, 363-406, 1976.

Farrell, W.E., Deformation of the Earth by surface loads, Rev. Geophys. and Space Phys., 10, 761-797, 1972.

Grafarend, E.W., Three-dimensional deformation analysis: global vector spherical harmonic and local finite element representation, Tectonophysics, 130, 337-359, 1986.

Lavallée, D., and G. Blewitt, Degree-1 Earth deformation from very long baseline interferometry, Geophys. Res. Lett., 29(20), 1967, doi:10.1029/2002GL015883, 2002.

Particle Data Group (Hagiwara K., et al.), 2002 review of particle physics, Phys. Rev. D 66, 010001, 2002.

Wahr, J.M., The effects of the atmosphere and oceans on the Earth's wobble. 1. Theory, Geophys. Journ. Roy. Astron. Soc., 70, 349-372, 1982. 